\title{
Declaración de las Sociedades Científicas Neumológicas Ibero-Latinoamericanas sobre los dispositivos electrónicos de liberación de nicotina
}

Las principales sociedades científicas de neumología y cirugía torácica de la región Iberolatinoamericana, tras las reuniones mantenidas en el seno de distintos congresos nacionales en los que se analizó la información publicada sobre los dispositivos electrónicos de liberación de nicotina (DELN), quieren hacer el siguiente manifiesto.

1.- El tratamiento más eficaz y seguro para ayudar a los fumadores a abandonar el consumo es la combinación de asesoramiento psicológico y de tratamiento farmacológico. Existen tres de tratamiento farmacológico con efectividad demostrada: terapia sustitutiva con nicotina (goma de mascar, parches, comprimidos, caramelos, inhaladores y spray), bupropión y vareniclina. Todos ellos utilizados a dosis y tiempo estándar son capaces de duplicar y hasta triplicar las posibilidades de dejar de fumar. ${ }^{1-3}$

2.- El análisis científico de los estudios realizados a la fecha demuestran que los DELN no han demostrado efectividad, o que es muy limitada, para dejar de fumar. ${ }^{4,5}$

3.- Los DELN no han demostrado seguridad. Existen múltiples estudios independientes que señalan efectos adversos a corto plazo. Además, no se puede descartar la aparición de efectos adversos a medio-largo plazo, hoy desconocidos por el corto período de observación. ${ }^{6-8}$ Por otro lado, se están promoviendo con agresivas campañas promocionales con identidad de marca con foco en jóvenes y adolescentes, de manera similar a las usadas por las tabacaleras en el siglo XX, con el objetivo de reclutar usuarios haciéndolos adictos a la nicotina.

4.- Por lo expuesto, afirmamos que los profesionales sanitarios no deben recomendar la utilización de este tipo de dispositivos en ningún caso.

5.- En fumadores con grandes dificultades para dejar de fumar o en aquellos en los que la utilización de los tratamientos a dosis y tiempo estándar han fracasado recomendamos de asesoramiento psicológico intenso más la prescripción de tratamiento farmacológico (terapia sustitutiva con nicotina, bupropión y vareniclina) a dosis altas, durante tiempo prolongado y en combinación. ${ }^{9}$

6.- Del análisis de diferentes estudios se observa que los dispositivos electrónicos de liberación de nicotina permiten la inhalación de otras substancias (saborizantes, cocaína, drogas sintéticas y derivados de cannabis, entre otras) que además del poder adictivo, suman nuevas toxicidades potenciales que pueden afectar adversamente sobre el aparato respiratorio. ${ }^{6-8}$

7.- La salud respiratoria demanda respirar aire limpio. La inhalación directa o de segunda mano de humo y/o aerosoles generados por DELN $\mathrm{u}$ otros dispositivos representa una amenaza a la salud respiratoria. Todas las estructuras que componen el aparato respiratorio están hechas y perfectamente dispuestas para cumplir con una de las principales necesidades que tenemos los seres humanos: la respiración del aire limpio y oxigenado, sin ella la vida no sería posible.

8.- Las sociedades de profesionales comprometidas con una respiración sana, alertamos a la población general que evite la utilización de cualquier tipo de dispositivo que le facilite la inhalación de sustancias extrañas cuya seguridad no haya sido comprobada científicamente.

\section{Bibliografía}

1.- FIORE MC, JAÉN CR, BAKER TB, BAILEY WC, BENOWITZ NL, CURRY SJ, et al. Treating Tobacco Use and Dependence: 2008 Update. Clinical Practice Guideline. Rockville, MD: U.S. Department of Health and Human Services. Public Health Service; 2008. Traducción al español: Jiménez Ruiz CA, Jaén CR (coordinadores de la traducción). Guía de tratamiento del tabaquismo. Sociedad Española de Neumología y Cirugía Torácica (SEPAR); 2010.

2.- ANTHENELLI RM, BENOWITZ NL, WEST R, ST AUBIN L, MCRAE T, LAWRENCE D, et al. Neuropsychiatric safety and efficacy of varenicline, bupropion, and nicotine patch in smokers with and without psychiatric disorders (EAGLES): a double-blind, randomized, placebo-controlled clinical trial. Lancet. 2016; 387(10037): 2507-20.

3.- HARTMANN-BOYCE J, CHEPKIN SC, YE W, 
BULLEN C, LANCASTER T. Nicotine replacement therapy versus control for smoking cessation. Cochrane Database Syst Rev. 2018; 5: CD000146.

4.- HARTMANN-BOYCE J, MCROBBIE H, BULLEN C, BEGH R, STEAD LF, HAJEK P. Electronic cigarettes for smoking cessation. Cochrane Database Syst Rev. 2016 Sep 14; 9: CD010216.

5.- HAJEK P, PHILLIPS-WALLER A, PRZULJ D, et al. A randomized trial of e-cigarettes versus nicotinereplacement therapy. N Engl J Med 2019; 380: 629-37.

6.- BALS R, BOYD J, ESPOSITO S, FORONJY R, HIEMSTRA PS, JIMÉNEZ-RUIZ CA, et al. Electronic cigarettes: a task force report from the European Respiratory Society. Eur Respir J. 2019; 31: 53(2).

7.- ZABERT G, GAGA M, JIMÉNEZ RUIZ C, BULJUVACICH D, GARCÍA G, VÁZQUEZ J, RAVARA S y BE-
LLO S. Cigarrillo electrónico y demás ENDS: posición de sociedades científicas respiratorias. Revista Americana de Medicina Respiratoria RAMR. 2019; vol 19: 2.

8.- SIGNES COSTA J, DE GRANDA ORIVE I, RAMOS PINEDO A, CAMARASA ESCRIG A, DE HIGES MARTÍNEZ E, RABADE C, CABRERA CESAR E, JIMÉNEZ RUIZ CA. Declaración oficial de la Sociedad Española de Neumología y Cirugía Torácica SEPAR sobre cigarrillos electrónicos e IQOS. Arch Bronconeumol. 2019. En prensa.

9.- JIMÉNEZ-RUIZ CA, ANDREAS S, LEWIS KE, TONNESEN P, VAN SCHAYCK CP, HAJEK P, et al. Statement on smoking cessation in COPD and other Pulmonary Disorders and in smokers with comorbidities who find it difficult to quit. Eur Respir J. 2015; 46(1): 61-79. 